\title{
Interpretation of the sonic hedgehog morphogen gradient by a temporal adaptation mechanism
}

\author{
Eric Dessaud ${ }^{1}$, Lin Lin Yang ${ }^{2} \dagger$, Katy Hill ${ }^{1}$, Barny Cox ${ }^{1}$, Fausto Ulloa ${ }^{1}$, Ana Ribeiro ${ }^{1}$, Anita Mynett ${ }^{1}$, \\ Bennett G. Novitch ${ }^{2} \uparrow \&$ James Briscoe ${ }^{1}$
}

\begin{abstract}
Morphogens act in developing tissues to control the spatial arrangement of cellular differentiation ${ }^{1,2}$. The activity of a morphogen has generally been viewed as a concentration-dependent response to a diffusible signal, but the duration of morphogen signalling can also affect cellular responses ${ }^{3}$. One such example is the morphogen sonic hedgehog ( $\mathrm{SHH})$. In the vertebrate central nervous system and limbs, the pattern of cellular differentiation is controlled by both the amount and the time of SHH exposure ${ }^{4-7}$. How these two parameters are interpreted at a cellular level has been unclear. Here we provide evidence that changing the concentration or duration of $\mathrm{SHH}$ has an equivalent effect on intracellular signalling. Chick neural cells convert different concentrations of SHH into time-limited periods of signal transduction, such that signal duration is proportional to $\mathrm{SHH}$ concentration. This depends on the gradual desensitization of cells to ongoing $\mathrm{SHH}$ exposure, mediated by the SHH-dependent upregulation of patched 1 (PTC1), a ligand-binding inhibitor of SHH signalling ${ }^{8}$. Thus, in addition to its role in shaping the SHH gradient $^{8-10}$, PTC1 participates cell autonomously in gradient sensing. Together, the data reveal a novel strategy for morphogen interpretation, in which the temporal adaptation of cells to a morphogen integrates the concentration and duration of a signal to control differential gene expression.
\end{abstract}

How both concentration and duration of morphogen signalling determine cell pattern is poorly understood ${ }^{1}$ (see Supplementary Fig. 1a). We focused on three transcription factors that respond to differential SHH signalling in progenitors of the neural tube. OLIG2 and NKX2.2, expressed in the ventral neural tube of chick (Fig. 1a), depend on $\mathrm{SHH}$ signalling for their expression ${ }^{4,11,12}$. In contrast, PAX7 expression is repressed by $\mathrm{SHH}$ signalling ${ }^{13}$ and is restricted to dorsal neural tube progenitors (Fig. 1a). We confirmed that these proteins respond to different levels of $\mathrm{SHH}$ signalling using an ex vivo assay of intermediate region naive neural plate explants ${ }^{14}$ (Fig. 1b-d). In agreement with previous studies ${ }^{13,15}$, changes in $\mathrm{SHH}$ concentration controlled the expression of these genes in a manner corresponding to their in vivo expression patterns (Fig. 1e). Similar gene expression responses were obtained by generating, in vivo, a gradient of GLI transcriptional activity ${ }^{16,17}$, the transcriptional effectors of $\mathrm{SHH}$ signalling. Furthermore, manipulation of the activity of the transmembrane protein smoothened (SMO), which transduces SHH signalling intracellularly ${ }^{18}$, was also sufficient to confer graded responses to neural cells (Supplementary Fig. 2).

We asked how the response of cells to $\mathrm{SHH}$ develops over time. We assayed intermediate region neural plate explants exposed to $\mathrm{SHH}$ for $6 \mathrm{~h}$ to $24 \mathrm{~h}$ (Fig. 1f, g and Supplementary Table 1). NKX2.2 induction was delayed compared to OLIG2, taking $>12 \mathrm{~h}$ compared to $\sim 6 \mathrm{~h}$ for OLIG2. Moreover, NKX2.2-inducing concentrations of $\mathrm{SHH}(\geq 2 \mathrm{nM}$ ) produced a transient expression of OLIG2 (Fig. 1f, g). Thus, during the first $12 \mathrm{~h}, \geq 1 \mathrm{nM}$ SHH generated similar amounts of OLIG2 induction (Supplementary Table 1). Only after $12 \mathrm{~h}$ were distinct responses apparent. By $18 \mathrm{~h}$, OLIG2 and NKX2.2 co-expression was evident in some progenitors treated with $\geq 2 \mathrm{nM}$ $\mathrm{SHH}$ (Fig. 1f). These results are consistent with the sequential onset of OLIG2 and NKX2.2 expression in vivo ${ }^{10,17}$. Furthermore, the explant data predict that, in vivo, NKX2.2 should be induced in cells that previously expressed OLIG2. Genetic lineage tracing in mice harbouring an Olig2 allele engineered to encode Cre recombinase confirmed this (Fig. 1h). Thus, compared to OLIG2, induction of NKX2.2 requires a higher concentration and longer duration of SHH exposure.

To investigate the reason for the temporal and concentration dependence of the response, we analysed the output of the SHH signal transduction pathway. We assayed GLI activity using a reporter plasmid $^{19}$ (GBS-Luc; see Fig. 2a and Methods). Taking advantage of the short half-life of luciferase $e^{20}(\sim 3 \mathrm{~h})$, we measured GBS-Luc activity every $6 \mathrm{~h}$. For concentrations of $\mathrm{SHH} \geq 1 \mathrm{nM}$, GLI activity was similar during the first $12 \mathrm{~h}$ (Fig. $2 \mathrm{a}$ ), the period when these concentrations induce OLIG2. Then, with the exception of the highest SHH concentration, GLI activity decreased over time, with a rate inversely proportional to SHH concentration (Fig. 2a). Notably, the time at which $1 \mathrm{nM}$ and $4 \mathrm{nM}$ SHH produced differences in the level of GLI activity corresponded to the detection of differences in gene expression (Fig. 1f, g and Supplementary Table 1). The data indicate, therefore, that cells become progressively desensitized to ongoing SHH signalling (Supplementary Fig. 1). Initially $1 \mathrm{nM}$ and $4 \mathrm{nM} \mathrm{SHH}$ produce similar levels of GLI activity, then the level of GLI activity begins to fall, with a higher rate of decrease in cells exposed to lower concentrations. This suggests a mechanism for gradient sensing in which 'temporal adaptation' to the ligand transforms the extracellular concentration of morphogen into a time-limited period of signal transduction, such that the duration of signalling is proportional to ligand concentration.

This model predicts that the response of cells relies not only on the level but also on the duration of intracellular signal transduction. To test this, we compared GLI activity and gene expression in neural cells containing an endogenous source of SHH. In explants consisting of SHH-producing notochord and floor plate together with ventral regions of the neural tube (hereafter called NVF explants), the expression of OLIG2 and NKX2.2 was induced sequentially, $12 \mathrm{~h}$ and $18 \mathrm{~h}$ after the start of culture, respectively (Fig. $2 \mathrm{f}-\mathrm{h}$ ). During this period the level of GLI activity remained approximately constant, confirming that the switch to NKX2.2 expression was not associated with an increase in GLI activity (Fig. 2b, $\mathrm{f}-\mathrm{h}$ ). To examine whether the maintenance of an OLIG2-expressing state depends on the downregulation 
of GLI activity, NVF explants were cultured for $12 \mathrm{~h}$ and then transferred to media containing cyclopamine, a small-molecule antagonist of SHH signalling (Supplementary Fig. 2). GLI activity and gene expression were monitored at $18 \mathrm{~h}$ (Fig. 2c-e, i). Addition of $200 \mathrm{nM}$ cyclopamine at $12 \mathrm{~h}$ resulted in a twofold decrease in GLI activity and a failure to induce NKX2.2 expression (Fig. 2c, e, i) without inhibiting OLIG2 expression. Furthermore, addition of $400 \mathrm{nM}$ cyclopamine inhibited GLI activity to background levels, leading to a complete loss of NKX2.2 expression and a decrease in the number of OLIG2-expressing cells (Fig. 2c, e, and data not shown). These data indicate that the duration of GLI activity is crucial for determining the cellular response to $\mathrm{SHH}$. To test the converse prediction of the model, we assayed the effect of extending the period of $\mathrm{SHH}$ signalling (Fig. 2j). Consistent with the model, prolonging $\mathrm{SHH}$ signalling resulted in peak OLIG2 and NKX2.2 induction at lower SHH concentrations after $48 \mathrm{~h}$ of exposure compared to $24 \mathrm{~h}$ and $36 \mathrm{~h}$ (Fig. 2j).

Two mechanisms that could account for the adaptation of cells to SHH signalling are the loss of a factor necessary for signal transduction or the induction of inhibitors of signal transduction, such as the SHH-binding protein PTC1 (refs 21-23). We assessed GLI activity in a time series of intermediate region neural plate explants in which $\mathrm{SHH}$ signalling was induced using purmorphamine, a small-molecule agonist of $\mathrm{SMO}^{24}$ (Fig. 3a and Supplementary Fig. 2). In contrast to the response of neural cells to $\mathrm{SHH}$ (Fig. 2a), even though a diminution in GLI activity was observed in treated cells, there was no correlation between the rate of decrease and the concentration of purmorphamine (Fig. 3a). This suggests that the profile of GLI activity evoked by SHH is shaped mainly by an adaptation mechanism acting upstream of SMO.

As PTC1 is a well-established inhibitor of SHH signalling ${ }^{8}$, we tested whether it is required cell autonomously for the temporal adaptation of cells to SHH. Small interfering RNAs (siRNAs) were used to block chick PTC1 (cPTC1) induction (Fig. 3b and Supplementary Figs 3-6). In ovo transfection of $c P T C 1$ siRNAs inhibited the upregulation of $c P T C 1$ but did not completely abolish its expression (Supplementary Fig. 4, and data not shown). As a result, and consistent with observations in mouse embryos containing an un-inducible allele of $\mathrm{Ptcl}^{10}$, ventralization of the neural tube, but no ligand-independent $\mathrm{SHH}$ signalling, was observed in siRNA-transfected embryos or in intermediate region neural plate explants (Supplementary Figs 3 and 5). We therefore assayed whether blocking CPTC1 upregulation altered
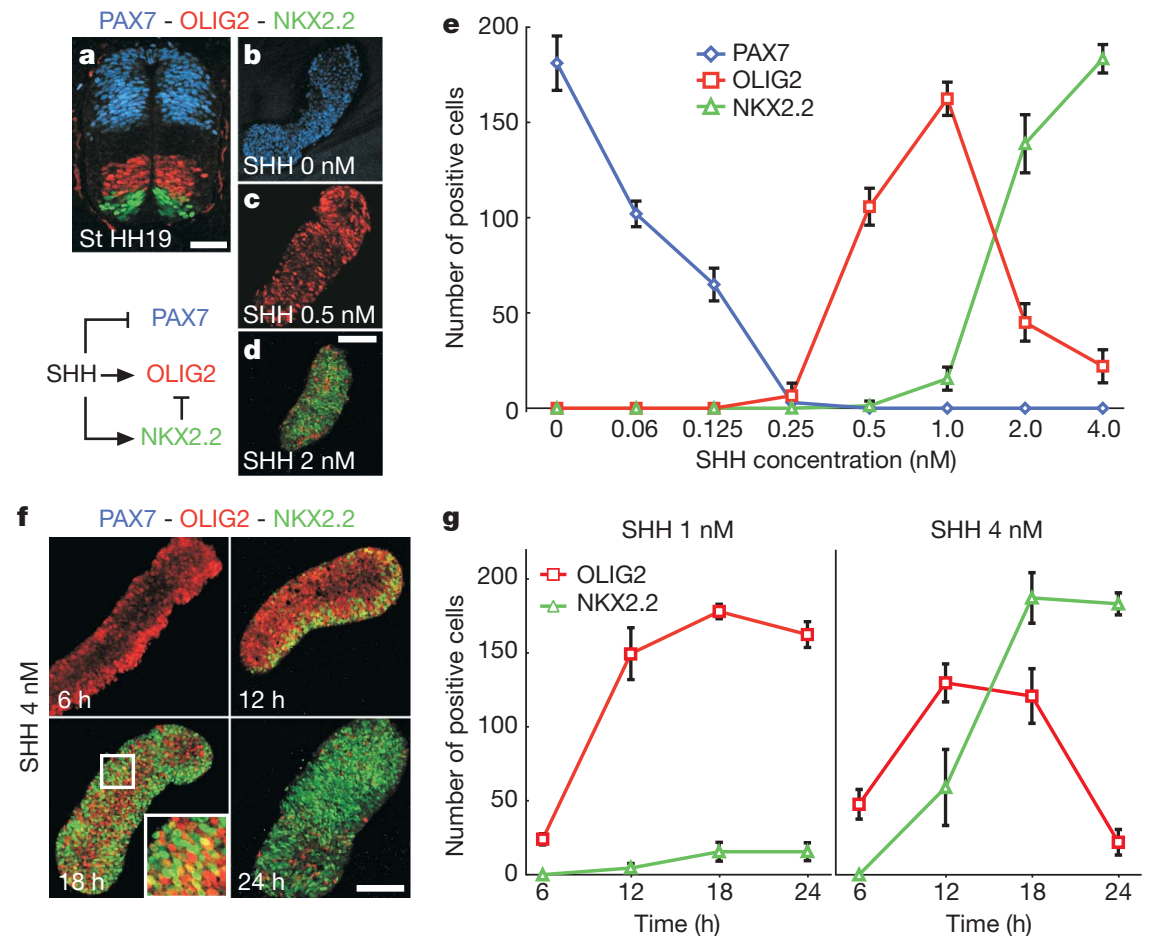

h

Olig2 2 re/+; ROSA26-flox-STOP-flox-lacZ
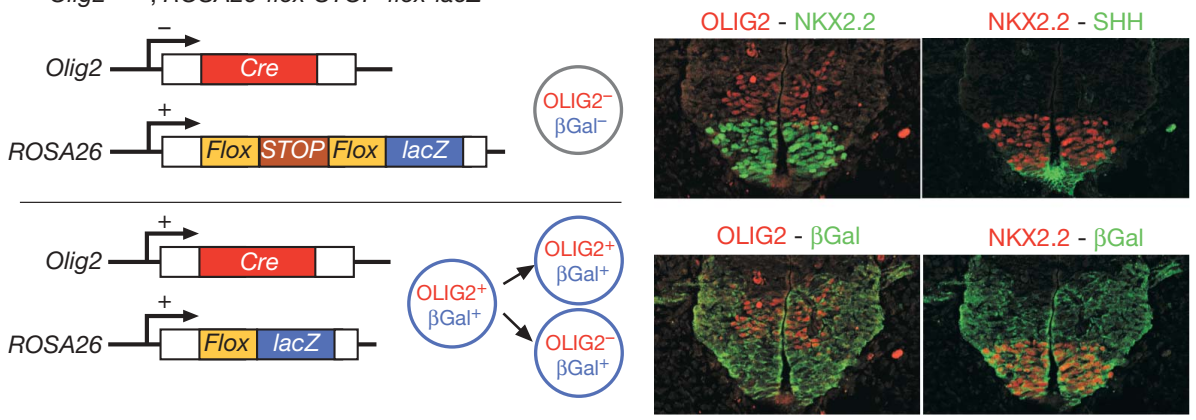

Figure 1 | The temporal and concentration dependence of the response of neural cells to SHH. a, PAX7, OLIG2 and NKX2.2 expression in HH stage 19 chick neural tube (scale bar, $50 \mu \mathrm{m}$ ). b-d, Intermediate region neural plate explants cultured for $24 \mathrm{~h}$ in the indicated concentrations of $\mathrm{SHH}$ and assayed simultaneously for the expression of PAX7, OLIG2 and NKX2.2. OLIG2 expression decreases as NKX2.2 is induced, consistent with the ability of NKX2.2 to repress OLIG2 ${ }^{12}$. e, Quantification of cells expressing PAX7, OLIG2 and NKX2.2 in intermediate region neural plate explants

( $n \geq 5$; number of cells per unit \pm s.d.). f, PAX7, OLIG2 and NKX2.2 expression in intermediate region neural plate explants grown with $4 \mathrm{nM}$ SHH for $6 \mathrm{~h}, 12 \mathrm{~h}, 18 \mathrm{~h}$ or $24 \mathrm{~h}$ (scale bar, $100 \mu \mathrm{m})$. g, Quantification of cells expressing OLIG2 and NKX2.2 in intermediate region neural plate explants ( $n \geq 5$; number of cells per unit \pm s.d.). $\mathbf{h}$, As well as OLIG $2^{+}$progenitors, $\mathrm{NKX} 2.2^{+}$progenitors express LacZ in Olig2 ${ }^{\text {Cre/+ }} ;$ ROSA26-flox-STOP-floxlacZ E10.5 mouse embryos. 
a
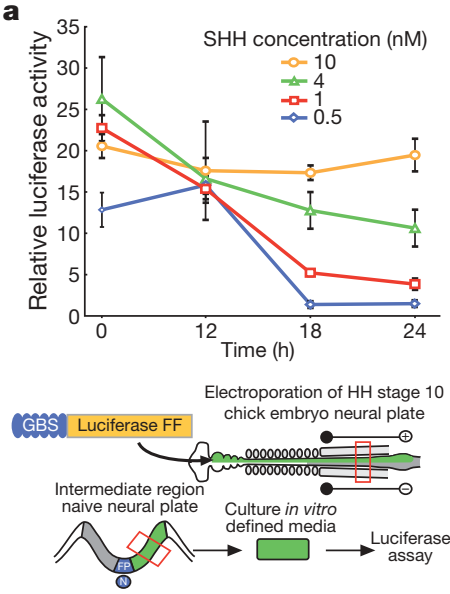

b

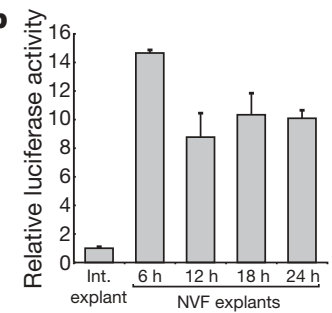

c

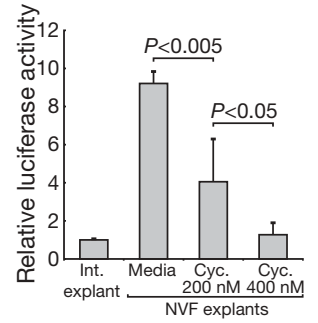

Figure 2 Sustained GLI activity is required for discrimination between OLIG2 and NKX2.2 gene induction. a, GLI activity (relative GLI activity \pm s.e.m.) measured with GBS-Luc in intermediate region neural plate explants treated with the indicated concentrations of SHH for the designated times. GLI activity is measured relative to untreated explants. b, GLI activity (relative GLI activity \pm s.e.m.) assayed in cells exposed to an endogenous source of SHH. GBS-Luc assays were performed using NVF explants

(d) compared to intermediate region neural plate explants, which lack SHH expression. c, Graph of relative GLI activity \pm s.e.m. according to conditions
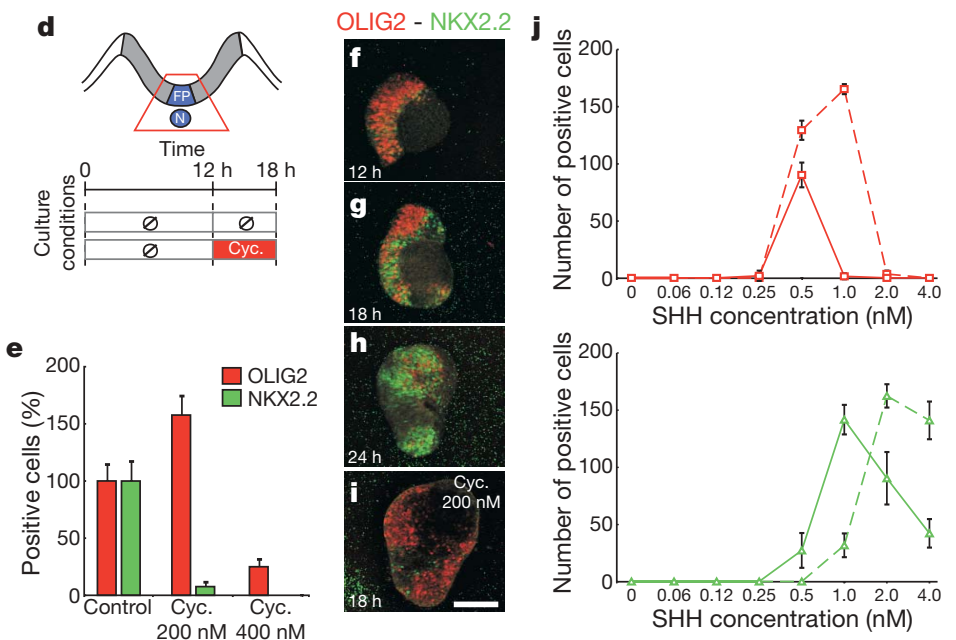

in d ( $P$-values from student's $t$-test). Cyc., cyclopamine. Decreasing the duration of GLI activity in NVF explants alters gene expression $(\mathbf{e}, \mathrm{i})$. e, Quantification of OLIG2 ${ }^{+}$and NKX2.2 $2^{+}$cells relative to control condition ( $18 \mathrm{~h}$ culture; \pm s.d.) in NVF explants. $\mathbf{f}-\mathbf{h}$, Temporal profile of NKX2.2 and OLIG2 expression in NVF explants. $\mathbf{j}$, Numbers of cells expressing OLIG2 (red) and NKX2.2 (green) in intermediate region neural plate explants ( $n \geq 5$; number of cells per unit \pm s.d.) exposed to the indicated concentrations of SHH for $36 \mathrm{~h}$ (dashed lines) and $48 \mathrm{~h}$ (solid lines).

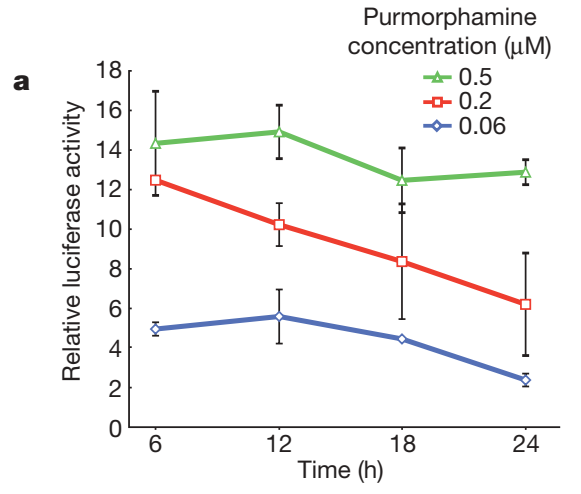

c

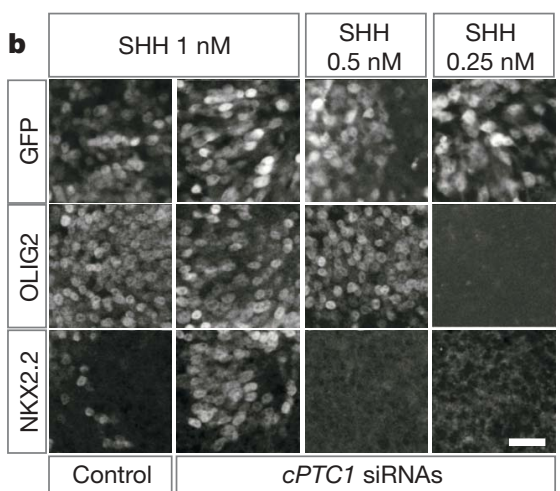

Figure 3 | A cell autonomous function for PTC1-mediated desensitization is required for gradient sensing. a, Temporal profile of GLI activity, measured with GBS-Luc, in intermediate region neural plate explants treated with the indicated concentrations of purmorphamine for the designated times (relative GLI activity \pm s.e.m.). b, Blocking cPTC1 upregulation disrupts the interpretation of high concentrations of SHH. Control or $C P T C 1$ siRNA electroporated intermediate region neural plate explants where cultured with the indicated concentrations of SHH for $24 \mathrm{~h}$ and assayed for NKX2.2 and OLIG2 (scale bar, $25 \mu \mathrm{m} ; n=10$ for control siRNA; $n=12$ for $c P T C 1$ siRNAs). c, A model for the morphogen patterning of a tissue divided into

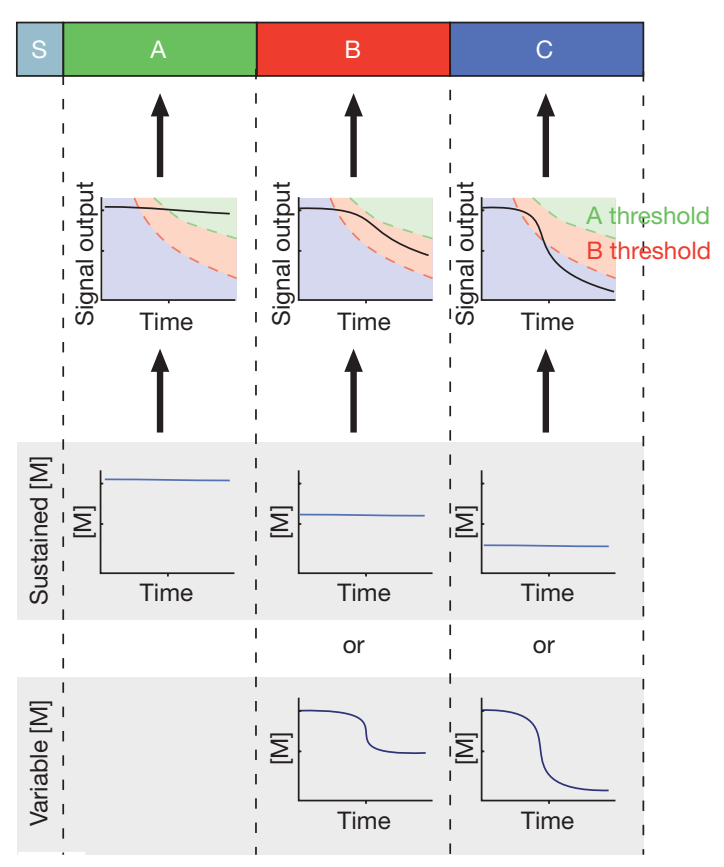

three domains characterized by differential gene expression (A, B and C). Graded morphogen signalling (M) from the source $(S)$ is transduced into an episode of signal transduction (signal output). Initially, each $M$ concentration $([\mathrm{M}])$ generates similar levels of signal output. However, signal output declines more rapidly in cells exposed to lower [M]. Similar profiles of signal output can be generated by a sustained $[\mathrm{M}]$ or by transient exposure to $\mathrm{M}$ (variable $[\mathrm{M}]$ ). This model illustrates how two different parameters of a graded morphogen - amount of ligand and duration of exposure-are transformed to an equivalent output by a responding cell. 
the response of cells to $\mathrm{SHH}$ exposure. Explants were transfected with control or $c$ PTC1 siRNAs and exposed to $0.25-1 \mathrm{nM}$ SHH. Transfection of control siRNAs did not change the gene expression profile induced by $\mathrm{SHH}$ (Fig. 3b). However, in contrast to control explants exposed to $1 \mathrm{nM} \mathrm{SHH}$ that predominately expressed OLIG2, increased numbers of NKX2.2-expressing cells were observed in $1 \mathrm{nM} \mathrm{SHH}$ treated intermediate region neural plate explants transfected with cPTC1 siRNAs. A similar result was obtained when siRNAs were transfected individually (Supplementary Fig. 6). The siRNA effect was dependent on $\mathrm{SHH}$ concentration: $c P T C 1$ siRNAs did not alter the response of intermediate region neural plate explants exposed to $<0.5 \mathrm{nM}$ SHH, indicating that the blockade of cPTC1 upregulation does not result in NKX2.2 induction in response to all concentrations of $\mathrm{SHH}$. These data indicate that gradient sensing requires upregulation of PTC1. This complements the previously identified ability of PTC1 to influence the spread of ligand by sequestering $\mathrm{SHH}$ protein ${ }^{8-10}$. Thus, upregulation of PTC1 by SHH has both cell autonomous and nonautonomous functions in morphogen interpretation.

Adaptation to extracellular signals has previously been proposed to permit the sensing and transduction of a signal over large concentration ranges ${ }^{25,26}$. This study indicates that the same strategy is used to measure the concentration of a ligand during developmental patterning. The progressive adaptation of cells to $\mathrm{SHH}$ transforms ligand exposure into periods of increased GLI activity, the duration of which is proportional to $\mathrm{SHH}$ concentration (Fig. 3c). This allows cells to integrate both the concentration and duration of a graded morphogen (Supplementary Fig. 1). The transduction of a morphogen concentration into a duration of transcriptional activity offers an alternative to the established mechanisms of graded signalling ${ }^{27,28}$, which explains how the amount and duration of $\mathrm{SHH}$ exposure determine similar cellular responses ${ }^{3,6,7}$.

\section{METHODS SUMMARY}

Neural plate explants, immunohistochemistry and in situ hybridization. Explants isolated from Hamburger and Hamilton (HH) stage 10 chick embryos were cultured as described ${ }^{13,14}$. Cyclopamine (Toronto Research Chemicals) and purmorphamine (Calbiochem) were dissolved in 100\% ethanol and DMSO, respectively. Reagents and protocols for immunohistochemistry and in situ hybridization have been described ${ }^{12-14,29}$.

Chick in ovo electroporation, luciferase assays and siRNAs. HH stage 10 embryos were electroporated in ovo and incubated for $2 \mathrm{~h}$ before dissecting explants. Luciferase assays with GBS-Luc ${ }^{19}$ were carried out as previously described ${ }^{17}$. RNA duplexes targeting chick PTC1 and, as a control, human lamin were from Dharmacon. These were co-electroporated in ovo with a plasmid expressing green fluorescent protein.

Olig2-Cre lineage tracing. Olig2-Cre mice were generated in the Jessell laboratory, Columbia University. Standard homologous recombination procedures were used to replace exon 2 of the Olig2 locus with Cre recombinase. Olig2-Crel + mice were mated with ROSA26 reporter mice ${ }^{30}$, and embryos analysed at E10.5.

Full Methods and any associated references are available in the online version of the paper at www.nature.com/nature.

\section{Received 6 September; accepted 3 October 2007.}

1. Gurdon, J. B. \& Bourillot, P. Y. Morphogen gradient interpretation. Nature 413, 797-803 (2001).

2. Wolpert, L. One hundred years of positional information. Trends Genet. 12, 359-364 (1996).

3. Pages, F. \& Kerridge, S. Morphogen gradients. A question of time or concentration? Trends Genet. 16, 40-44 (2000).

4. Briscoe, J. \& Ericson, J. Specification of neuronal fates in the ventral neural tube. Curr. Opin. Neurobiol. 11, 43-49 (2001).

5. Ahn, S. \& Joyner, A. L. Dynamic changes in the response of cells to positive hedgehog signaling during mouse limb patterning. Cell 118, 505-516 (2004).

6. Harfe, B. D. et al. Evidence for an expansion-based temporal Shh gradient in specifying vertebrate digit identities. Cell 118, 517-528 (2004).

7. Yang, Y. et al. Relationship between dose, distance and time in Sonic Hedgehogmediated regulation of anteroposterior polarity in the chick limb. Development 124, 4393-4404 (1997).
8. Chen, Y. \& Struhl, G. Dual roles for patched in sequestering and transducing Hedgehog. Cell 87, 553-563 (1996).

9. Briscoe, J., Chen, Y., Jessell, T. M. \& Struhl, G. A hedgehog-insensitive form of patched provides evidence for direct long-range morphogen activity of sonic hedgehog in the neural tube. Mol. Cell 7, 1279-1291 (2001).

10. Jeong, J. \& McMahon, A. P. Growth and pattern of the mammalian neural tube are governed by partially overlapping feedback activities of the hedgehog antagonists patched 1 and Hhip1. Development 132, 143-154 (2005).

11. Lei, Q. et al. Wnt signaling inhibitors regulate the transcriptional response to morphogenetic Shh-Gli signaling in the neural tube. Dev. Cell 11, 325-337 (2006).

12. Novitch, B. G., Chen, A. I. \& Jessell, T. M. Coordinate regulation of motor neuron subtype identity and pan-neuronal properties by the bHLH repressor Olig2. Neuron 31, 773-789 (2001).

13. Ericson, J. et al. Pax6 controls progenitor cell identity and neuronal fate in response to graded Shh signaling. Cell 90, 169-180 (1997).

14. Yamada, T., Pfaff, S. L., Edlund, T. \& Jessell, T. M. Control of cell pattern in the neural tube: motor neuron induction by diffusible factors from notochord and floor plate. Cell 73, 673-686 (1993).

15. Ericson, J., Morton, S., Kawakami, A., Roelink, H. \& Jessell, T. M. Two critical periods of Sonic Hedgehog signaling required for the specification of motor neuron identity. Cell 87, 661-673 (1996).

16. Lei, Q., Zelman, A. K., Kuang, E., Li, S. \& Matise, M. P. Transduction of graded Hedgehog signaling by a combination of Gli2 and Gli3 activator functions in the developing spinal cord. Development 131, 3593-3604 (2004).

17. Stamataki, D., Ulloa, F., Tsoni, S. V., Mynett, A. \& Briscoe, J. A gradient of Gli activity mediates graded Sonic Hedgehog signaling in the neural tube. Genes Dev. 19, 626-641 (2005).

18. Lum, L. \& Beachy, P. A. The Hedgehog response network: sensors, switches, and routers. Science 304, 1755-1759 (2004).

19. Sasaki, H., Hui, C., Nakafuku, M. \& Kondoh, H. A binding site for Gli proteins is essential for HNF-3 $\beta$ floor plate enhancer activity in transgenics and can respond to Shh in vitro. Development 124, 1313-1322 (1997).

20. Thompson, J. F., Hayes, L. S. \& Lloyd, D. B. Modulation of firefly luciferase stability and impact on studies of gene regulation. Gene 103, 171-177 (1991).

21. Chuang, P. T. \& McMahon, A. P. Vertebrate Hedgehog signalling modulated by induction of a Hedgehog-binding protein. Nature 397, 617-621 (1999).

22. Goodrich, L. V., Johnson, R. L., Milenkovic, L., McMahon, J. A. \& Scott, M. P. Conservation of the hedgehog/patched signaling pathway from flies to mice: induction of a mouse patched gene by Hedgehog. Genes Dev. 10, 301-312 (1996).

23. Marigo, V. \& Tabin, C. J. Regulation of patched by sonic hedgehog in the developing neural tube. Proc. Natl Acad. Sci. USA 93, 9346-9351 (1996).

24. Sinha, S. \& Chen, J. K. Purmorphamine activates the Hedgehog pathway by targeting Smoothened. Nature Chem. Biol. 2, 29-30 (2006).

25. Baker, M. D., Wolanin, P. M. \& Stock, J. B. Signal transduction in bacterial chemotaxis. Bioessays 28, 9-22 (2006).

26. Matthews, H. R. \& Reisert, J. Calcium, the two-faced messenger of olfactory transduction and adaptation. Curr. Opin. Neurobiol. 13, 469-475 (2003).

27. Bourillot, P. Y., Garrett, N. \& Gurdon, J. B. A changing morphogen gradient is interpreted by continuous transduction flow. Development 129, 2167-2180 (2002).

28. Saha, K. \& Schaffer, D. V. Signal dynamics in Sonic hedgehog tissue patterning. Development 133, 889-900 (2006).

29. Briscoe, J., Pierani, A., Jessell, T. M. \& Ericson, J. A homeodomain protein code specifies progenitor cell identity and neuronal fate in the ventral neural tube. Cell 101, 435-445 (2000).

30. Soriano, P. Generalized lacZ expression with the ROSA26 Cre reporter strain. Nature Genet. 21, 70-71 (1999).

Supplementary Information is linked to the online version of the paper at www.nature.com/nature.

Acknowledgements We thank $\mathrm{H}$. Sasaki for the GBS-Luc reporter construct. We are grateful to S. Kales, B. Han and M. Mendelsohn for help in generating Olig2-Cre mice and to A. Gould, J. P. Vincent and V. Ribes for discussions. This work was supported by the MRC (UK). B.G.N. is supported by grants from the Whitehall Foundation, the March of Dimes Foundation, the NINDS and the University of Michigan Biomedical Scholars Program. Olig2-Cre mice were generated with support from HHMI and NINDS to T. M. Jessell. E.D. is supported by EMBO and Marie Curie Fellowships.

Author Contributions E.D. and J.B. conceived the work, performed the experiments, analysed data and wrote the manuscript. L.L.Y and B.G.N. analysed Olig2-Cre mice. K.H., B.C., F.U., A.R. and A.M. provided materials and technical assistance.

Author Information Reprints and permissions information is available at www.nature.com/reprints. Correspondence and requests for materials should be addressed to J.B. (james.briscoe@nimr.mrc.ac.uk). 


\section{METHODS}

Neural plate explant culture. Neural plate tissue was isolated from Hamburger and Hamilton $(\mathrm{HH})$ stage 10 chick embryos and cultured as described ${ }^{14}$. Explants consisted of the intermediate region or the notochord, floor plate and adjacent ventral region (NVF). SHH protein was generated as described ${ }^{15}$. Concentration of $\mathrm{SHH}$ was determined by comparison with previous batches. Cyclopamine (Toronto Research Chemicals) and purmorphamine (Calbiochem) were dissolved in 100\% ethanol and DMSO, respectively. Quantifications of PAX7, OLIG2 and NKX2.2 expressing cells were performed on representative unit squares (containing $200 \pm 20$ cells) in intermediate region neural plate explants ( $n \geq 5$ explants for each data point).

Immunohistochemistry and in situ hybridization. Antibody reagents and protocols have been described ${ }^{12-14,29}$. The monoclonal antibodies against PAX6 and PAX7 were obtained from the Developmental Studies Hybridoma Bank (University of Iowa). Rabbit Nkx2.2 antibody $^{31}$ (T. Jessell) was used at 1:5,000 and guinea-pig Olig2 antibody ${ }^{12}$ was used at 1:10,000. Mouse antibody for Tuj1 (Covance Research) and goat antibody for Sox2 (ISL) were used at 1:1,000 and 1:500, respectively. Sheep antibody for GFP (Biogenesis) and rabbit antibody for GFP (Invitrogen) were used at 1:1,000. Analysis was carried out using a Leica TCS SP2 confocal microscope or a Zeiss Axioplan 2 and processed with Adobe Photoshop 7.0 software (Adobe Systems). In situ hybridization was performed as described $^{32}$ using a chick PTC1 probe $^{33}$ labelled with DIG-labelling reagents (Roche Diagnostics) and filtered NBT/BCIP substrate (Sigma) in 0.5\% Tween (Sigma).

Generation of $\boldsymbol{c P T C 1}$ siRNAs. RNA duplexes targeting $c P T C 1$ mRNA were ordered in 2' deprotected, annealed and desalted form (Option A4, http:// www.dharmacon.com). The RNA duplexes used in this study are: $c P T C 1$ siRNA 1 sense $5^{\prime}$-UGAGAGCUAUCUGCAAUAAUU-3'; $c P T C 1$ siRNA 2 sense 5' -AUAUGGACCAGAUUAUAGAUU-3' ${ }^{\prime}$; PTC1 siRNA 3 sense $5^{\prime}$-CUAAUGUACUCACAACAGAUU-3' . siRNA (target sequence 5' -AACUGGACUUCCAGAAGAACA-3') against human lamin was used as control (Dharmacon).

Chick in ovo electroporation and luciferase assay in intermediate region explants. $\mathrm{HH}$ stage 10 embryos were electroporated and incubated for $2 \mathrm{~h}$ before dissecting intermediate region neural plate explants. For luciferase assays, the firefly-luciferase reporter gene carrying eight copies of the GLI-binding site from the FoxA2 floor plate enhancer (GBS-Luc) was used ${ }^{19}$. Chick embryos were coelectroporated with GBS-Luc and CMV-Renilla luciferase normalization plasmid (Promega). Assays were carried out on explants as previously described ${ }^{17}$. Explants were homogenized in passive lysis buffer on ice and measurement of firefly and Renilla luciferase activities was performed using the Dual Luciferase Reporter Assay System (Promega). Each point corresponds to triplicates of 7-10 explants. Short interfering RNAs were electroporated at $2.5 \mu \mathrm{g} \mu \mathrm{l}^{-1}$ final concentration. The neural tube of embryos was co-injected with a plasmid expressing GFP to control for electroporation efficiency.

Olig2-Cre lineage tracing. Standard homologous recombination procedures were used to replace exon 2 of the Olig2 locus with a gene encoding a nuclearlocalized form of Cre recombinase. Details of the gene-targeting strategy are available on request. Olig2-Cre/+ mice were mated with ROSA26 reporter mice $^{30}$, and embryos were collected at E10.5 and subjected to immunohistochemical analysis as described above.

31. Briscoe, J. et al. Homeobox gene Nkx2.2 and specification of neuronal identity by graded Sonic hedgehog signalling. Nature 398, 622-627 (1999).

32. Dessaud, E., Salaun, D., Gayet, O., Chabbert, M. \& deLapeyriere, O. Identification of lynx2, a novel member of the ly-6/neurotoxin superfamily, expressed in neuronal subpopulations during mouse development. Mol. Cell. Neurosci. 31, 232-242 (2006).

33. Pearse, R. V. II, Vogan, K. J. \& Tabin, C. J. Ptc1 and Ptc2 transcripts provide distinct readouts of Hedgehog signaling activity during chick embryogenesis. Dev. Biol. $239,15-29$ (2001). 